University of New Hampshire

University of New Hampshire Scholars' Repository

Space Science Center

Institute for the Study of Earth, Oceans, and

Space (EOS)

2009

\title{
Statistical Properties of Gamma-Ray Burst Polarization
}

\author{
Kenji Toma \\ Pennsylvania State University - Main Campus \\ Takanori Sakamoto \\ NASA Goddard Space Flight Ctr. \\ Bing Zhang \\ University of Nevada, Las Vegas \\ J E. Hill \\ NASA Goddard Space Flight Ctr. \\ Mark L. McConnell \\ University of New Hampshire - Main Campus, mark.mcconnell@unh.edu
}

See next page for additional authors

Follow this and additional works at: https://scholars.unh.edu/ssc

Part of the Astrophysics and Astronomy Commons

\section{Recommended Citation}

Statistical Properties of Gamma-Ray Burst Polarization Toma, K. and Sakamoto, T. and Zhang, B. and Hill, J. E. and McConnell, M. L. and Bloser, P. E. and Yamazaki, R. and loka, K. and Nakamura, T., AIP Conference Proceedings, 1133, 434-436 (2009), DOI:http://dx.doi.org/10.1063/1.3155943

This Conference Proceeding is brought to you for free and open access by the Institute for the Study of Earth, Oceans, and Space (EOS) at University of New Hampshire Scholars' Repository. It has been accepted for inclusion in Space Science Center by an authorized administrator of University of New Hampshire Scholars' Repository. For more information, please contact Scholarly.Communication@unh.edu. 


\section{Authors}

Kenji Toma, Takanori Sakamoto, Bing Zhang, J E. Hill, Mark L. McConnell, Peter F. Bloser, Ryo Yamazaki, Kunihito loka, and Takashi Nakamura 


\section{AIP $\mid$ proceedings}

\section{Statistical Properties of GammaRay Burst Polarization}

K. Toma, T. Sakamoto, B. Zhang, J. E. Hill, M. L. McConnell, P. E. Bloser, R. Yamazaki, K. loka, and T. Nakamura

Citation: AIP Conference Proceedings 1133, 434 (2009); doi: 10.1063/1.3155943

View online: http://dx.doi.org/10.1063/1.3155943

View Table of Contents: http://scitation.aip.org/content/aip/proceeding/aipcp/1133?ver=pdfcov

Published by the AIP Publishing

\section{Articles you may be interested in}

The Addition of Duration Does Not Improve The Luminosity Relations for Gamma Ray Bursts

AIP Conf. Proc. 1133, 476 (2009); 10.1063/1.3155956

Theoretical Implications of Thermal Emission from GammaRay Bursts

AIP Conf. Proc. 1133, 317 (2009); 10.1063/1.3155909

Quasiblackbody component in the prompt emission of gammaray bursts

AIP Conf. Proc. 1133, 312 (2009); 10.1063/1.3155908

GammaRay Burst Polarization

AIP Conf. Proc. 1040, 186 (2008); 10.1063/1.2981543

Polarized Gravitational Waves from GammaRay Bursts

AIP Conf. Proc. 686, 84 (2003); 10.1063/1.1629418 


\title{
Statistical Properties of Gamma-Ray Burst Polarization
}

\author{
K. Toma ${ }^{*}$, T. Sakamoto ${ }^{\dagger, * *}$, B. Zhang ${ }^{\ddagger}$, J. E. Hill ${ }^{\dagger, 8}$, M. L. McConnell ${ }^{\text {I }}$, P. E. Bloser ${ }^{\text {g }}$, \\ R. Yamazakill, K. Ioka ${ }^{\dagger \dagger}$ and T. Nakamura \\ ${ }^{*}$ Department of Astronomy and Astrophysics, Pennsylvania State University, 525 Davey Lab, University Park, \\ PA 16802, USA \\ ${ }^{\dagger}$ CRESST and NASA Goddard Space Flight Center, Greenbelt, MD 20771, USA \\ ${ }^{* *}$ Joint Center for Astrophysics, University of Maryland, Baltimore County, 1000 Hilltop Circle, Baltimore, MD \\ 21250, USA \\ Department of Physics and Astronomy, University of Nevada Las Vegas, Las Vegas, NV 89154, USA \\ ${ }^{\S}$ Universities Space Research Association, 10211 Wincopin Circle, Suite 500, Columbia, MD, 21044-3432, USA \\ ISpace Science Center, University of New Hampshire, Durham, NH 03824, USA \\ "Department of Physics, Hiroshima University, Higashi-Hiroshima, Hiroshima 739-8526, Japan \\ \# Theory Division, KEK (High Energy Accelerator Research Organization), 1-1 Oho, Tsukuba 305-0801, Japan \\ \# Department of Physics, Kyoto University, Kyoto 606-8502, Japan
}

\begin{abstract}
The emission mechanism and the origin and structure of magnetic fields in gamma-ray burst (GRB) jets are among the most important open questions concerning the nature of the central engine of GRBs. In spite of extensive observational efforts, these questions remain to be answered and are difficult or even impossible to infer with the spectral and lightcurve information currently collected. Polarization measurements will lead to unambiguous answers to several of these questions. Recent developments in X-ray and $\gamma$-ray polarimetry techniques have demonstrated a significant increase in sensitivity enabling several new mission concepts, e.g. POET (Polarimeters for Energetic Transients), providing wide field of view and broadband polarimetry measurements. If launched, missions of this kind would finally provide definitive measurements of GRB polarizations. We perform Monte Carlo simulations to derive the distribution of GRB polarizations in three emission models; the synchrotron model with a globally ordered magnetic field (SO model), the synchrotron model with a locally random magnetic field (SR model), and the Compton drag model (CD model). The results show that POET, or other polarimeters with similar capabilities, can constrain the GRB emission models by using the statistical properties of GRB polarizations. In particular, the ratio of the number of GRBs for which the polarization degrees can be measured to the number of GRBs that are detected $\left(N_{m} / N_{d}\right)$ and the distributions of the polarization degrees (I) can be used as the criteria. If $N_{m} / N_{d}>30 \%$ and $\Pi$ is clustered between 0.2 and 0.7 , the SO model will be favored. If instead $N_{m} / N_{d}<15 \%$, then the SR or CD model will be favored. If several events with $\Pi>0.8$ are observed, then the CD model will be favored.
\end{abstract}

Keywords: $\gamma$-ray sources; $\gamma$-ray bursts; Radiation mechanisms; polarization

PACS: $98.70 . \mathrm{Rz}, 01.30 . \mathrm{Cc}, 95.30 . \mathrm{Gv}$

\section{INTRODUCTION}

In spite of extensive observational and theoretical efforts, several key questions concerning the nature of the central engines of the relativistic jets and the jets themselves of gamma-ray bursts (GRBs) remain poorly understood. In fact, some of these questions are very diffi cult or even impossible to answer with the spectral and lightcurve information currently collected. On the other hand, polarization information, if retrieved, would lead to unambiguous answers to these questions. In particular, polarimetric observations of GRBs can address the following: (i) It is unclear whether a globally structured magnetic fi eld plays essential roles on the jet dynamics and the burst emission. (ii) It is unclear what is the most relevant emission mechanism; synchrotron, Comptonization, thermal emission, or else. (iii) The distribution of the jet opening angles and the observer's viewing angles, and the structure of the jet are not well understood.

Recently there has been an increasing interest in the measurement of X-ray and $\gamma$-ray polarization, and the observational techniques can now achieve signifi cant sensitivity in the relevant energy band. Several polarimetry mission concepts, such as POET, are being planned. The POET concept has two polarimeters, GRAPE (60-500 keV) and LEP (2-15 keV) both of which have wide fi elds of view. If launched, missions of this type would provide the first defi nitive detection of the polarization of GRB prompt emission. This would enable the discussion of the statistical properties of the polarization degree and polarization spectra, which will give us diagnostic information on the emission mechanism of GRBs and the nature of the GRB jets that cannot be obtained from current spectra and

CP1133, Gamma Ray Bursts, $6^{\text {th }}$ Huntsville Symposium

edited by C. Meegan, N. Gehrels, and C. Kouveliotou

(C) 2009 American Institute of Physics 978-0-7354-0670-4/09/\$25.00 
lightcurve observations.

So far it has been shown that similarly high levels of linear polarization can be obtained in several GRB prompt emission models; the synchrotron model with a globally ordered magnetic fi eld, the synchrotron model with a smallscale random magnetic fi eld $[1,2,3]$, and the Compton drag model $[4,5]$. Thus the detections of GRB prompt emission polarization would support these three models. In this paper, we show that these models can be distinguished by their statistical properties of observed polarizations. We performed detailed calculations of the distribution of polarization degrees by including realistic spectrum of GRB prompt emission and assuming realistic distributions of the physical parameters of GRB jets, and show that POET, or other polarimeters with similar capabilities, can constrain the GRB emission models. We use the limits of POET for GRB detection and polarization measurements as realistic and fi ducial limits. For detailed descriptions of our theoretical calculations and the properties of POET, see $[6,7,8]$.

\section{THEORETICAL MODELS}

We calculate the linear polarization for instantaneous emission from a thin spherical shell moving radially outward with a bulk Lorentz factor $\gamma \gg 1$ and an opening angle $\theta_{j}$. The comoving-frame emissivity has the functional form of $j_{v^{\prime}}^{\prime I}=A_{0} f\left(v^{\prime}\right) \delta\left(t^{\prime}-t_{0}^{\prime}\right) \delta\left(r^{\prime}-r_{0}^{\prime}\right)$, where $A_{0}$ is the normalization which may depend on direction in the comoving frame and other physical quantities of the shell and $f\left(v^{\prime}\right)$ represents the spectral shape. A prime represents the physical quantities in the comoving frame. The delta functions describe the instantaneous emission at $t=t_{0}$ and $r=r_{0}$. The normalization, $A_{0}$, has units of erg cm $\mathrm{cm}^{-2} \mathrm{str}^{-1} \mathrm{~Hz}^{-1}$. Using the spherical coordinate system $(r, \theta, \phi)$ in the lab frame, where $\theta=0$ is the line of sight, we obtain the spectral fluence:

$$
I_{v}=\frac{1+z}{d_{L}^{2}} \int d \phi \int d(\cos \theta) r_{0}^{2} \frac{A_{0} f\left(v^{\prime}\right)}{\gamma^{2}(1-\beta \cos \theta)^{2}},
$$

where $z$ and $d_{L}$ are the redshift and the luminosity distance of the source, respectively, and $v^{\prime}=(1+z) v \gamma(1-\beta \cos \theta)$. The integration is performed within the jet cone, so that it depends on the viewing angle $\theta_{v}$, i.e., the angle between the jet axis and the line of sight. The corresponding Stokes parameters of the local emission (i.e., the emission from a given point on the shell) are given by $j_{v^{\prime}}^{\prime O}=j_{v^{\prime}}^{\prime I} \Pi_{0}^{\prime} \cos \left(2 \chi^{\prime}\right)$ and $j_{v^{\prime}}^{\prime U}=j_{v^{\prime}}^{\prime I} \Pi_{0}^{\prime} \sin \left(2 \chi^{\prime}\right)$, where $\Pi_{0}^{\prime}$ and $\chi^{\prime}$ are the polarization degree and position angle of the local emission measured in the comoving frame, respectively. The Stokes parameters of the emission from the whole shell can be obtained by integrating those of the local emission similarly to the intensity $I_{v}$ :

$$
\left\{\begin{array}{c}
Q_{v} \\
U_{v}
\end{array}\right\}=\frac{1+z}{d_{L}^{2}} \int d \phi \int d(\cos \theta) r_{0}^{2} \frac{A_{0} f\left(v^{\prime}\right)}{\gamma^{2}(1-\beta \cos \theta)^{2}} \Pi_{0}\left\{\begin{array}{c}
\cos (2 \chi) \\
\sin (2 \chi)
\end{array}\right\} .
$$

The polarization degree is Lorentz invariant, i.e., $\Pi_{0}^{\prime}=\Pi_{0}$. The position angle $\chi$ is calculated by taking account of the Lorentz transformation of the electromagnetic waves, and it is measured from a fi xed direction, which we choose to be the direction from the line of sight to the jet axis. Then by calculating $\{I, Q, U\}=\int_{v_{1}}^{v_{2}} d v\left\{I_{v}, Q_{v}, U_{v}\right\}$, we obtain the time-averaged linear polarization in the given wavebands $\left[v_{1}, v_{2}\right]$ :

$$
\Pi=\frac{\sqrt{Q^{2}+U^{2}}}{I} .
$$

We consider synchrotron and Compton drag (CD) mechanisms for the GRB prompt emission. In the synchrotron case, the magnetic $f$ eld consists of a globally ordered $\mathbf{f i}$ eld, $\mathbf{B}_{\mathrm{rd}}$, and small-scale random $\mathrm{fi}$ eld, $\mathbf{B}_{\mathrm{fnd}}$, i.e., $\mathbf{B}=$ $\mathbf{B}_{\text {ord }}+\mathbf{B}_{\mathrm{rnd}}$. The fi eld $\mathbf{B}_{\text {ord }}$ may originate from the central engine, while $\mathbf{B}_{\mathrm{rnd}}$ may be produced in the emission region itself. Here we consider two extreme cases; synchrotron model with an ordered fi eld (SO), in which $B_{\text {ord }}^{2} \gg\left\langle B_{\text {rnd }}^{2}\right\rangle$, and a synchrotron model with a random fi eld (SR), in which $B_{\text {ord }}^{2} \ll\left\langle B_{\text {rnd }}^{2}\right\rangle$. For the SO model, in particular, we assume a toroidal magnetic fi eld. We give $A_{0}, f\left(v^{\prime}\right), \Pi_{0}$, and $\chi$ as functions of $(\theta, \phi)$ for each model, and calculate the linear polarization for given parameters $\gamma, \theta_{j}, \boldsymbol{\theta}_{v}$, and $z$.

\section{STATISTICAL PROPERTIES}

In this section we show the results of our Monte Carlo simulation of the GRB prompt emission polarization. We generated 10,000 GRB jets with Lorents factor, $\gamma$, and opening angle, $\theta_{j}$, and a random viewing angle for each 

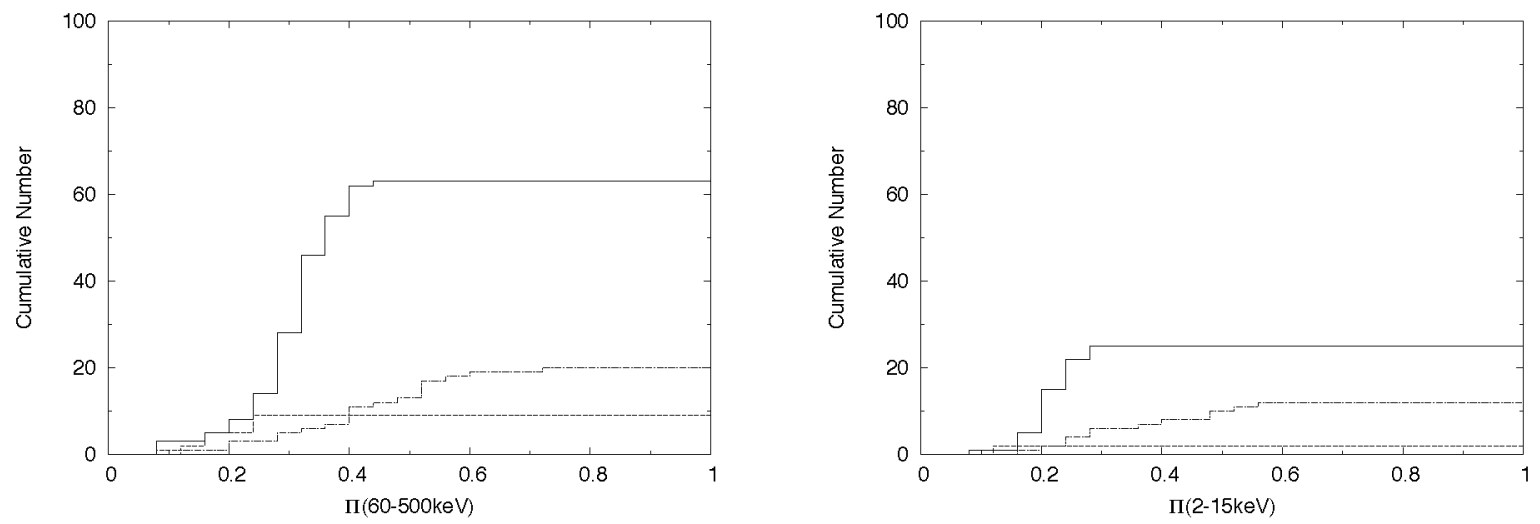

FIGURE 1. The cumulative distribution of $\Pi$ that can be measured by GRAPE (left) and LEP (right) in the SO (solid), SR (dashed), and CD (dot-dashed) models in which the number of detectable bursts is 200. The adopted parameters are $\gamma=100, q_{1}=$ $0.5, q_{2}=-2.0, \alpha=-0.2, \beta=1.2$, and $T=20 \mathrm{~s}$.

jet according to the probability distribution of $\sin \theta_{v} d \theta_{\nu} d \phi$. We give the values of the model parameters so that the observed fluences and peak energies of simulated bursts are consistent with the data obtained with the HETE-2 satellite. The distributions of $\gamma$ and $\theta_{j}$ for GRB jets are highly uncertain. We fi $\times \gamma=100$. We assume the distribution of $\theta_{j}$ as $f\left(\theta_{j}\right) d \theta_{j} \propto \theta_{j}^{q_{1}} d \theta_{j}$ for $0.001 \leq \theta_{j} \leq 0.02$ and $\propto \theta_{j}^{q_{2}} d \theta_{j}$ for $0.02 \leq \theta_{j} \leq 0.2$, where $q_{1}=0.5$ and $q_{2}=-2.0$. We also $\mathrm{fi}$ x the values of low and high spectral indices of the burst, $\alpha=-0.2$ and $\beta=1.2$ respectively, and the duration $T=20 \mathrm{~s}$.

We calculate the linear polarization, $\Pi$, for each GRB jet to obtain the polarization distribution. We obtain the distribution of polarization that can be measured, by using the MDP (Minimum Detectable Polarization) values of $P O E T$. We interpret the simulated events with $\Pi>M D P$ as ' $\Pi$-measurable events'. Figure 1 shows the cumulative distribution of $\Pi$ that can be measured by GRAPE and LEP in the SO, SR, and CD models. We have set the number of detectable events $N_{d}=200$. In the SO model, the number of $\Pi$-measurable bursts is $N_{m}>60$, and the cumulative distribution of measurable $\Pi$ varies rapidly at $0.3<\Pi<0.4$ for the GRAPE band. In the SR model, $N_{m}<10$, and the maximum polarization is $\Pi_{\max }<0.4$, In the CD model, $N_{m}<30$, and $\Pi_{\max }<0.8$.

[6] derive more general conclusion for $\gamma \geq 100, q_{1} \geq 0.5$, and $q_{2} \geq-3.0$, taking account of realistic distributions of $\alpha$ and $\beta$. In conclusion, we can constrain the emission mechanism of GRBs by using the cumulative distribution obtained by GRAPE. If $N_{m} / N_{d}>30 \%$, the SR and CD models may be ruled out, and in this case if the measured polarizations are clustered at $0.2<\Pi<0.7$, the SO model will be favored. If $N_{m} / N_{d}<15 \%$, the SO model may be ruled out, but we cannot distinguish between the SR and CD models with different distributions of $\left(\gamma \theta_{j}\right), \alpha$, and $\beta$. If several bursts with $\Pi>0.8$ are detected, however, the CD model which includes adequate number of small $\left(\gamma \theta_{j}\right)$ bursts will be favored.

\section{REFERENCES}

1. J. Granot, ApJ 596, L17-L21 (2003), arXiv: astro-ph/0306322.

2. M. Lyutikov, V. I. Pariev, and R. D. Blandford, ApJ 597, 998-1009 (2003), arXiv : astro-ph/0305410.

3. E. Nakar, T. Piran, and E. Waxman, Journal of Cosmology and Astro-Particle Physics 10, 5-+ (2003), arXiv: astro-ph/0307290.

4. D. Lazzati, E. Rossi, G. Ghisellini, and M. J. Rees, MNRAS 347, L1-L5 (2004), arXiv : astro-ph/0309038.

5. D. Eichler, and A. Levinson, ApJ 596, L147-L150 (2003), arXiv : astro-ph/0306360.

6. K. Toma, T. Sakamoto, B. Zhang, J. E. Hill, M. L. McConnell, P. F. Bloser, R. Yamazaki, K. Ioka, and T. Nakamura, ArXiv e-prints (2008), 0812.2483 .

7. J. E. Hill, M. L. McConnell, P. Bloser, J. Legere, J. Macri, J. Ryan, S. Barthelmy, L. Angelini, T. Sakamoto, J. K. Black, D. H. Hartmann, P. Kaaret, B. Zhang, K. Ioka, T. Nakamura, K. Toma, R. Yamazaki, and X. Wu, "POET: POlarimeters for Energetic Transients," in American Institute of Physics Conference Series, 2008, vol. 1065 of American Institute of Physics Conference Series, pp. 331-337.

8. P. F. Bloser, J. S. Legere, M. L. McConnell, J. R. Macri, C. M. Bancroft, T. P. Connor, and J. M. Ryan, ArXiv e-prints (2008), 0812.0782 . 